\title{
Association between fish consumption, long chain omega 3 fatty acids, and risk of cerebrovascular disease: systematic review and meta-analysis
}

\author{
Rajiv Chowdhury research associate ${ }^{1}$, Sarah Stevens public health registrar ${ }^{5}$, Donal Gorman PhD \\ candidate $^{1}$, An Pan research associate ${ }^{2}$, Samantha Warnakula PhD candidate ${ }^{1}$, Susmita Chowdhury \\ research associate ${ }^{6}$, Heather Ward research fellow ${ }^{4}$, Laura Johnson epidemiologist ${ }^{1}$, Francesca \\ Crowe nutritional epidemiologist ${ }^{3}$, Frank B Hu professor ${ }^{2}$, Oscar H Franco professor ${ }^{17}$
}

${ }^{1}$ Department of Public Health and Primary Care, University of Cambridge, UK; ${ }^{2}$ Department of Nutrition, Harvard School of Public Health, Boston, USA; ${ }^{3}$ Cancer Epidemiology Unit, Nuffield Department of Clinical Medicine, University of Oxford, UK; ${ }^{4}$ Department of Epidemiology and Biostatistics, School of Public Health, Imperial College London, UK; ${ }^{5}$ Public Health Directorate, NHS Midlands and East, Fulbourn, Cambridge, UK; ${ }^{6} \mathrm{Public}$ Health Genomics Foundation, Cambridge, UK; ${ }^{7}$ Department of Epidemiology, Erasmus MC, University Medical Center Rotterdam, Rotterdam, Netherlands baseline long chain omega 3 fatty acids with the bottom thirds for circulating biomarkers was 1.04 (0.90 to 1.20) and for dietary exposures was $0.90(0.80$ to 1.01$)$. In the randomised controlled trials the relative risk for cerebrovascular disease in the long chain omega 3 supplement compared with the control group in primary prevention trials was 0.98 (0.89 to 1.08) and in secondary prevention trials was 1.17 (0.99 to 1.38). For fish or omega 3 fatty acids the estimates for ischaemic and haemorrhagic cerebrovascular events were broadly similar. Evidence was lacking of heterogeneity and publication bias across studies or within subgroups.

Conclusions Available observational data indicate moderate, inverse associations of fish consumption and long chain omega 3 fatty acids with cerebrovascular risk. Long chain omega 3 fatty acids measured as circulating biomarkers in observational studies or supplements in primary and secondary prevention trials were not associated with cerebrovascular disease. The beneficial effect of fish intake on cerebrovascular risk is likely to be mediated through the interplay of a wide range of nutrients abundant in fish.

\section{Introduction}

Fish consumption is considered one of the key components of a cardioprotective diet. ${ }^{1}$ Current cardiovascular guidelines ${ }^{23}$ for healthy individuals encourage consumption of a variety of fish, preferably oily types, at least twice a week. Cold water oily fish 
and fish oil are also the most common dietary sources of long chain omega 3 fatty acids, a group of polyunsaturated fats that primarily include eicosapentaenoic acid and docosahexaenoic acid. ${ }^{4}$ Studies show that regular consumption of both these fats as supplements may reduce arrhythmias, endothelial dysfunction, circulating triglyceride levels, and inflammation. ${ }^{56}$ Such findings have prompted clinical guidelines to recommend the use of these nutrients in people with pre-existing coronary heart disease or high blood levels of triglycerides. ${ }^{78}$ Whether, or to what extent, current recommendations for consumption of fish or long chain omega 3 fatty acids may apply to cerebrovascular diseases is, however, unclear. Observational evidence on consumption of fish and omega 3 fatty acid is inconsistent for cerebrovascular outcomes. ${ }^{9}$ Likewise, despite a growing body of experimental evidence on eicosapentaenoic acid and docosahexaenoic acid supplements, the results for cardiovascular prevention are conflicting. Whereas earlier trials reported moderate protective effects on coronary heart disease and sudden cardiac death outcomes, ${ }^{10}{ }^{11}$ recent large scale primary and secondary prevention trials failed to show the efficacy of supplementation with long chain fatty acids in reducing cardiovascular diseases. ${ }^{12}{ }^{13}$ Therefore, whether supplementing with long chain omega 3 fatty acids could specifically help prevent stroke, remains elusive.

Several studies have investigated the intake of fish and long chain omega 3 fatty acids in relation to cerebrovascular disease ${ }^{14-21}$ However, these studies varied in methodology (for example, self reported dietary intake of omega 3 fatty acids versus circulating biomarkers versus supplements), scientific rigour (for example, sufficient power and duration of follow-up), or the ability to evaluate associations in greater detail (for example, by stroke subtypes). Furthermore, previous meta-analyse ${ }^{22-24}$ dealing with these hypotheses did not assess important differences in the exposure type, such as white versus fatty fish consumption, within clinically relevant subgroups (for example, average intake or stroke subtypes); and did not systematically compare observational evidence in the context of experimental data in a single investigation.

We systematically reviewed and meta-analysed available studies to quantify the associations of fish consumption with total and cause specific cerebrovascular disease in prospective cohort studies; examined associations of dietary and circulating levels of long chain omega 3 fatty acids with cerebrovascular diseases in observational studies; and evaluated the potential effects of their supplementations on future cerebrovascular events in randomised controlled trials.

\section{Methods}

We carried out an electronic search using primarily Medline, supplemented by searches of Embase, BIOSIS, and the Science Citation Index databases, for studies published before September 2012, without any language restriction (see supplementary file for details of the search strategy). Potentially eligible studies were those that had reported associations of fish (defined as fish and other seafood) or omega 3 fatty acids consumption, circulating omega 3 fatty acids, and omega 3 fatty acid supplements or dietary fish interventions with cerebrovascular disease (defined as any fatal or non-fatal ischaemic stroke, haemorrhagic stroke, cerebrovascular accident, or transient ischaemic attack). In the computer based searches we combined search terms related to the exposure (for example, fish, fatty acids, omega 3 fatty acids) and outcomes of interest (stroke, cerebrovascular disorders, etc). We considered prospective cohort studies to be eligible for inclusion if they had at least one year of follow-up and involved either general populations (participants not selected on the basis of pre-existing disease) or people at high risk of cardiovascular disease (those selected on the basis of high risk factors for cardiovascular disease or pre-existing cardiovascular disease, or both). Randomised intervention studies were eligible for inclusion if they assessed the effects of long chain omega 3 fatty acids supplements or dietary fish consumption in adults; collected "hard" cerebrovascular disease endpoints, such as fatal or non-fatal ischaemic stroke, or fatal or non-fatal haemorrhagic stroke; and followed participants for at least one year. Two independent reviewers screened the titles, abstracts, and full texts of the initially identified studies to determine eligibility. Disagreements were resolved through consensus or consultation with a third author. To identify additional publications we searched the reference lists of all retrieved studies.

\section{Data collection process and data items}

Two independent reviewers used a predesigned data abstraction form to extract relevant information from the selected studies. The form included questions on study size, study design, baseline population (general populations or those at high risk of cardiovascular disease), geographical location, year of baseline survey, age range of participants at baseline, duration of follow-up, reported degree of adjustment for potential confounders (defined as " + " when relative risks were adjusted for age and sex, "++" after further adjustment for conventional vascular risk factors (for example, smoking status, history of diabetes, and hypertension), and "+++" when further adjusted for other additional variables (for example, social class or total energy intake), type and numbers of cerebrovascular disease outcomes, and reported relative risks for each outcome. Where appropriate we extracted information on type of fish (white or fatty), amount consumed daily, dietary assessment tool (dietary questionnaire, defined as food frequency or diet history questionnaires; diet records, defined as all open ended instruments such as 24 hour recall and food diaries), blinding status, and type and composition of supplement or placebo. In the case of multiple publications, we abstracted the most up to date or most comprehensive information.

\section{Quality evaluation assessment}

Two independent investigators evaluated the quality of the included studies by using a modified scoring system based on MOOSE, QUATSO, and STROBE guidelines. ${ }^{25-27}$ This allowed a total score from 0 to 6 points ( 6 representing the highest quality), with 1 point allocated if a study provided a study rationale; used appropriate selection criteria; employed a validated questionnaire (dietary studies) or assay method (blood based studies), or involved adequate blinding (randomised controlled trials); collected outcomes not solely based on self report; reported findings adjusted for age, sex, body mass index, and smoking status; and additionally adjusted for other covariates, such as social status and other nutrients.

\section{Statistical analysis}

To limit potential biases, the analyses involved only comparisons within studies (participants being directly compared only within each cohort). To enable a consistent approach to analyses, where appropriate, we transformed risk estimates from each prospective cohort study to enable comparisons between the top and bottom third of the population's baseline distribution of exposure values, using methods previously described. ${ }^{28}$ Briefly, we transformed log risk estimates assuming a normal distribution, with the 
comparison between top and bottom thirds being equivalent to 2.18 times the log risk ratio for a 1 standard deviation increase (or equivalently as 2.18/2.54 times the log risk ratio for a comparison of extreme quarters). We calculated standard errors of the log risk ratios using published confidence limits and then standardised them in the same way. Hazard and odds ratios were assumed to approximate the same measure of relative risks. Using a random effects model that included between study heterogeneity, we calculated summary relative risks by pooling the study specific estimates. Where studies reported relative risks with differing degrees of adjustment for other risk factors, we used the maximum adjusted estimate. Dose-response meta-analysis of fish consumption and cerebrovascular risk was done using methods previously reported, ${ }^{24}{ }^{29}$ which facilitated the calculation of a pooled relative risk across studies with a common unit of comparison within studies (the relative risk associated with a two incremental serving a week in this review), assuming a linear dose-response relation. Assessments for standardised categories of fish consumption (2-4 times a week and $\geq 5$ times a week) and cerebrovascular risk compared with a reference category ( $\leq 1$ a week) were based on combining comparable relative risk estimates across studies using random effects meta-analyses. ${ }^{22}$ As reference groups of the included studies varied (that is, from 0 to $\leq 1$ times/week), in this analysis, we defined " $\leq 1$ /week" as the reference category assuming that all reported reference exposures represent an equally low level of intake. Consistency of findings across studies was assessed by standard $\chi^{2}$ tests and the $\mathrm{I}^{2}$ statistic. ${ }^{30}$ Heterogeneity was quantified by comparing results from studies grouped according to prespecified study level characteristics using metaregression. Evidence of publication bias was assessed using funnel plots and the Egger test. ${ }^{31}$ All statistical tests were two sided and used a significance level of $\mathrm{P}<0.05$. Analyses were done using Stata release 11 .

\section{Results}

The search strategy identified 4343 unique citations. After the initial screening, based on titles and abstracts, 447 articles remained for further evaluation. Following detailed assessments, 402 articles were excluded (fig $1 \Downarrow$ ). Overall, 45 articles based on 38 unique studies met the inclusion criteria and were included in the meta-analysis (see supplementary file). All relevant studies identified were published in the English language. In aggregate, the included studies comprised 794000 unique participants and 34817 incident cerebrovascular disease outcomes from 15 countries (table $\Downarrow$ and supplementary tables).

\section{Fish consumption and cerebrovascular risk}

Information on fish consumption and cerebrovascular disease were available in 21 prospective cohort studies, totalling 675 048 participants and 25320 incident cerebrovascular events (see supplementary table 1). All 21 studies were based on general populations. Seven involved participants from Europe, seven from North America, and seven from the Asia-Pacific region. Six studies included only men, three only women, and the rest both sexes. The relative risk of cerebrovascular disease for standardised categories of fish intake, typically adjusted for several conventional risk factors, for $2-4$ versus $\leq 1$ servings a week was 0.94 (95\% confidence interval 0.90 to 0.98 ) and for $\geq 5$ versus $\leq 1$ servings a week was 0.88 ( 0.81 to 0.96 ) (based on 18 and eight studies, respectively; fig $2 \Downarrow$ and supplementary figs 1 and 2). There was no evidence of heterogeneity across studies $\left(\mathrm{I}^{2}=20-22 \%, \mathrm{P}>0.05\right.$ for both, see supplementary figs 1 and 2). In the dose-response meta-analysis (18 studies), an increment of two servings a week of any fish was associated with a $4 \%$ (95\% confidence interval $1 \%$ to $7 \%$ ) reduced risk of cerebrovascular disease (fig 2 and supplementary fig 3 ). For all 21 studies the relative risk when comparing participants in the highest with the lowest category of fish intake was $0.88(0.84$ to 0.93 , see supplementary fig 4). In a subset of studies (62 799 participants) the corresponding relative risk for white fish types was 1.03 (0.90 to 1.19$)$ and for fatty fish types was $0.84(0.72$ to 0.98 , see supplementary fig 5 ).

\section{Comparison with evidence from long chain omega 3 fatty acid studies}

Fourteen prospective studies reported on long chain omega 3 fatty acids in relation to risk of cerebrovascular disease, involving 305119 participants and 5374 cerebrovascular outcomes recorded during an average follow-up ranging from four to 30 years (see supplementary table 2). Ten studies reported on intake of dietary long chain omega 3 fatty acid, whereas four were based on circulating blood compositions of omega 3. Five studies involved participants from Europe, four from North America, and five from the Asia-Pacific region. Seven studies concerned both sexes, five only men, and two only women. All cohorts included healthy populations at baseline. Fig $3 \Downarrow$ and supplementary figs 6 and 7 present the relative risks for cerebrovascular disease for participants in the top compared with bottom third of baseline long chain omega 3 fatty acid and fish consumption. Relative risks for long chain omega 3 fatty acids measured as circulating biomarkers and self reported dietary exposures were 1.04 (95\% confidence interval 0.90 to 1.20$)$ and 0.90 (0.80 to 1.01$)$, respectively. The corresponding relative risk in the top compared with bottom third of baseline fish consumption (11 studies; 366787 participants) was 0.91 (0.86 to 0.97$)$. Similar results were obtained for ischaemic and haemorrhagic stroke events (fig 3 and supplementary figs 8 and 9).

\section{Effects of long chain omega 3 fatty acid supplements on cerebrovascular risk}

Twelve randomised controlled trials totalling 62040 participants reported on long chain omega 3 fatty acids supplementation and cerebrovascular disease (see supplementary table 3 ). No study assessed the effects of any dietary fish interventions. Most of these randomised controlled trials (nine out of 12) were done in Europe. Ten trials included participants with previous cardiovascular disease, whereas two concerned populations without any pre-existing cardiovascular disease at baseline. Participants in the intervention arm on average consumed 1.8 $\mathrm{g}$ of long chain omega 3 fatty acids daily (range 0.4-6.0 g/day), where an oil capsule was the principle form of supplementation. After an average follow-up of 3.0 (range 1.0-4.7 years) years, a total of 800 cerebrovascular events occurred among participants in the intervention group compared with 763 events in the control group, with a pooled relative risk of 1.03 (95\% confidence interval 0.94 to 1.12 ; fig $4 \Downarrow$ ). No evidence of heterogeneity was found among these randomised controlled trials $\left(\mathrm{I}^{2}=3.5 \%, \mathrm{P}=0.41\right.$, also see supplementary fig 10$)$. The corresponding pooled relative risk for primary prevention trials (two studies) was 0.98 (0.89 to 1.08) and for secondary prevention trials (10 studies) was 1.17 (0.99 to 1.38 , fig 4 and supplementary fig 10). The relative risks were broadly similar across baseline population and for cause specific cerebrovascular outcomes based on studies with available data (fig 4 and supplementary fig 10). 


\section{Stratified and sensitivity analyses}

The overall associations observed in prospective studies for consumption of fish and long chain omega 3 fatty acids remained broadly consistent when these studies were grouped by several characteristics at study level. For instance, the overall relative risk of cerebrovascular disease when comparing the highest with the lowest category of fish intake was 0.87 (95\% confidence interval 0.82 to 0.92$)$ and for studies with higher and lower average fish consumption was 0.89 (0.78 to 1.01) (P for heterogeneity $>0.05$; see supplementary fig 11$)$. Similarly, comparing studies with higher versus lower average consumption of long chain omega 3 fatty acids yielded relative risks of 0.93 (0.81 to 1.07 ) and 0.85 (0.72 to 1.01$)$, respectively ( $\mathrm{P}$ for heterogeneity $>0.05$, also see supplementary fig $12 \mathrm{a}$ ). For consumption of both fish and omega 3 fatty acids in cohort studies, the magnitude of the relative risks of cerebrovascular disease for women seemed larger than those for men. In the randomised controlled trials there was also no clear evidence of heterogeneity according to several trial characteristics (for example, amount and type of intervention, blinding status, trial size, or duration of follow-up), except for some evidence that larger studies tended to yield more extreme relative risks (see supplementary fig 12b). In both observational and experimental studies, subgroup analyses indicated no geographical variation for the associations.

\section{Analysis of publication bias}

There was no evidence of publication bias across prospective cohort studies of fish or long chain omega 3 fatty acid consumption and the randomised controlled trials included in this review ( $\mathrm{P}>0.05$ in Egger's asymmetry test for all, also see supplementary fig 13).

\section{Discussion}

This review found that higher fish consumption is moderately but significantly associated with a reduced risk of incident cerebrovascular disease. By contrast, dietary, circulating biomarkers and supplements of long chain omega 3 fatty acids were not significantly associated with risk of cerebrovascular disease. The combined estimates remained similar for cause specific cerebrovascular events. The associations for long chain omega 3 fatty acids were consistent across people with and without pre-existing cardiovascular disease-indicating a potential lack of benefit for either primary or secondary prevention of cerebrovascular disease. These findings therefore suggest that single nutrients may have limited effects on chronic disease outside of their original food sources.

The results observed in our review for fish consumption and cerebrovascular risk may have several alternative explanations. Firstly, it is possible that the potential benefit of fish consumption could in addition to long chain omega 3 fatty acids be attributed to a wider array of nutrients (and their interaction) that are abundant in fish. For example, fish are also a good source of vitamins D and B complex, which have been linked to inverse cerebrovascular risk. ${ }^{32}{ }^{33}$ In addition, essential amino acids and trace elements in fish (for example, taurine, ${ }^{34} 35$ arginine,${ }^{36}$ selenium,${ }^{37}$ calcium,${ }^{38}$ magnesium,${ }^{39}{ }^{40}$ potassium, ${ }^{41}$ and iodine ${ }^{42}$ ) may have potentially favourable vascular effects. Secondly, the positive impact of fish could be explained by a concomitant reduction in intake of foods detrimental to cerebrovascular health, such as red meat. Adjustment for confounding by other dietary factors is often inadequate and rarely accounts for such "protein substitution effect." ${ }^{43}$ None the less, in a recent analysis based on two large cohorts in the
United States, a 17\% reduction in cerebrovascular risk was observed when red meat intake was replaced with fish. ${ }^{43}$ Thirdly, higher fish consumption may simply be an indicator of a healthier dietary pattern or higher socioeconomic status, which themselves are associated with conventional vascular determinants and outcomes. ${ }^{1644}$ Although our subgroup analyses by levels of adjustment were consistent with the overall estimates, this may not fully address the potential issue of residual confounding, as individual characteristics such as baseline vascular drug use (antihypertensives, statins, aspirin, etc) or level of adherence (for instance for omega 3 supplements in randomised controlled trials) were generally unavailable in the studies included in the meta-analysis. The differences observed for the associations between white and fatty fish may be explained by the distinct ways in which the two foods are typically prepared. Studies that contributed to these analyses were exclusively based on the United Kingdom, ${ }^{17}$ where dietary questionnaires for the white fish items usually include battered and deep fried white fish; and in Sweden, where frying is the most common cooking method for white fish. ${ }^{15}$ This systematic difference in the preparation of white fish, in contrast with that of oily fish, could explain the null effect because potentially detrimental fats (such as trans fatty acids) are added during the cooking process. ${ }^{45}$

The discordant results observed for long chain omega 3 fatty acids compared with overall fish intake may also have various potential explanations. Firstly, food composition tables are often incomplete such that the long chain omega 3 fatty acid content of foods is unknown, which may lead to underestimation of the true intake of long chain omega 3 fatty acids. For example, only $65 \%$ of all foods in the US Department of Agriculture food composition database reportedly have a value for their omega 3 fatty acid content. ${ }^{46}$ Additionally, these databases may not account for all variations in the omega 3 fatty acid content of fish from different environments ${ }^{47}$ (for example, cooking methods, dietary patterns, or farm raised food versus wild sources). ${ }^{7}$ Secondly, self reported dietary questionnaires typically seek information on fish group rather than on specific fish types (for instance, oily fish versus salmon). Considering the substantial differences in quantity of omega 3 fatty acids between marine species, ${ }^{48}$ this inaccurate grouping of fish may lead to potential poor allocation of nutrient composition to food and overall misclassification of omega 3 fatty acid intake, which may attenuate the associations. Thirdly, although fish intake generally correlates highly with circulating blood omega 3 fatty acid levels, in the general Western population only $20-25 \%$ of the variation in such levels might be explained by fish consumption alone. ${ }^{49}$ This may be responsible, at least in part, for the inconsistent results between observational studies concerning fish and long chain omega 3 fatty acids. Fourthly, in our analyses circulating biomarkers of long chain omega 3 fatty acids were not associated with cerebrovascular disease. Although blood fatty acid levels are not affected by limitations in the dietary instruments or composition databases, these biomarkers only reflect exposures over the previous few weeks compared with a prolonged assessment that is more relevant to predict chronic disease risk. ${ }^{50}$ Finally, none of the randomised controlled trials were designed to investigate cerebrovascular disease as the primary outcome of interest. Therefore, competing risk events such as coronary heart disease may have altered the probability of cerebrovascular outcomes and impeded many subsequent events being considered thus limiting sufficiently powerful analyses. Furthermore, discrepant findings on omega 3 fatty acids in observational studies and randomised controlled trials may be partly explained by the inherent differences 
between baseline populations in the two designs- that is, generally healthy people compared with people with previous vascular disease or higher cardiovascular disease risk factors, respectively.

\section{Comparison with previous studies}

Findings of this updated meta-analysis generally concur and further extend the findings of previous reviews in several important ways. Our meta-analysis reinforces earlier results by including several recently published large scale prospective cohort studies. Our analyses on prospective dietary fish studies, for example, involved twice as many cerebrovascular events as the two previous reviews combined. ${ }^{22}{ }^{24}$ Similarly, analyses on long chain omega 3 fatty acids based on data from observational studies and trials had about 10 times as many stroke events as the previous analysis. ${ }^{23}$ The current review also evaluates the potential effects of long chain omega 3 supplements on cerebrovascular risk by comparing observational evidence in the context of experimental data, systematically in a single investigation. Additionally, it comprehensively records a wide range of clinically relevant subgroups to carry out the most detailed exploration of potential heterogeneity than previously reported.

\section{Implications of findings}

Our findings may have several implications. They reinforce a potentially modest beneficial role of fish intake in the cause of cerebrovascular disease. Such an advantage was less evident for long chain omega 3 fatty acids in both observational studies and interventions targeting primary and secondary stroke prevention. Our findings are in line with current dietary guidelines (that is, to encourage fish consumption for all; and intake of fish oils, preferably from oily fish, to people with pre-existing or at high risk of coronary heart disease) and favour propositions that the future nutritional guidelines should be principally "food based." ${ }^{11}$ They also underscore scientific gaps in the experimental evidence, specifically the lack of studies involving healthy populations and interventions targeting fish intake rather than using supplements, which may have different mechanistic effects. Additionally, adequately powered data from trials will be essential to investigate reliably the apparent higher risk of cerebrovascular disease observed in the secondary prevention trials of long chain omega 3 fatty acid supplements, and potential sex specific associations across observational studies.

\section{Strengths and limitations of this review}

The strengths and limitations of this review merit consideration. We included data from almost 800000 participants from 15 countries. There was limited evidence of heterogeneity and publication bias across studies or within subgroups. In the absence of individual patient level data, we used standardised risk estimates to allow consistent comparisons and we examined several clinically relevant characteristics to reduce potential heterogeneity. However, the current review was limited by an overall lack of available data on the cause specific cerebrovascular outcomes. For example, only a few studies reported more than 1000 ischaemic stroke events, whereas most involved analyses primarily on composite cerebrovascular events. Furthermore, even in aggregate, fewer than 2000 cerebrovascular events were available in the randomised controlled trials and none of them were based on healthy populations.

\section{Conclusions}

Available observational data demonstrate moderate, inverse associations of fish and long chain omega 3 fatty acids consumption with risk of cerebrovascular events. However, there was no evidence for similar inverse associations with cerebrovascular disease for long chain omega 3 fatty acids measured as circulating biomarkers in observational studies, or supplements in primary and secondary prevention trials. The beneficial effect of fish intake on cerebrovascular risk might be mediated through a complex interplay among a wide range of nutrients commonly found in fish.

Contributors: OHF and RC conceived the study. RC, SS, and DG did the analyses. SS, RC, DG, SW, SC, HW, and OHF did the literature searches and data extraction. RC, SS and OHF wrote the manuscript. $\mathrm{AP}, \mathrm{FBH}, \mathrm{FC}$, and $\mathrm{LJ}$ contributed to the initial revision of the manuscript. $\mathrm{RC}, \mathrm{OHF}, \mathrm{FC}, \mathrm{LJ}, \mathrm{AP}$, and FBH contributed to the critical revision of the manuscript before publication. SS and DG contributed equally to the study. OHF is the guarantor.

Funding: $\mathrm{RC}$ is recipient of a Gates Cambridge PhD scholarship, DG and $\mathrm{SW}$ are supported by funding from MRC studentships, and OHF is the recipient of a grant from Pfizer Nutrition to establish a new centre on aging research focused on nutrition and lifestyle.

Competing interests: All authors have completed the ICMJE uniform disclosure form at www.icmje.org/coi_disclosure.pdf (available on request from the corresponding author) and declare: no support from any organisation for the submitted work; no financial relationships with any organisations that might have an interest in the submitted work in the previous three years; and no other relationships or activities that could appear to have influenced the submitted work.

Ethical approval: Not required.

Data sharing: No additional data available.

1 Mozaffarian D, Appel LJ, Van HL. Components of a cardioprotective diet: new insights. Circulation 2011;123:2870-91

2 Gidding SS, Lichtenstein AH, Faith MS, Karpyn A, Mennella JA, Popkin B, et al. Implementing American Heart Association pediatric and adult nutrition guidelines: a scientific statement from the American Heart Association Nutrition Committee of the Council on Nutrition, Physical Activity and Metabolism, Council on Cardiovascular Disease in the Young, Council on Arteriosclerosis, Thrombosis and Vascular Biology, Council on Cardiovascular Nursing, Council on Epidemiology and Prevention, and Council for High Blood Pressure Research. Circulation 2009;119:1161-75.

3 Graham I, Atar D, Borch-Johnsen K, Boysen G, Burell G, Cifkova R, et al. European guidelines on cardiovascular disease prevention in clinical practice: executive summary: Fourth Joint Task Force of the European Society of Cardiology and Other Societies on Cardiovascular Disease Prevention in Clinical Practice (Constituted by representatives of nine societies and by invited experts). Eur Heart J 2007;28:2375-414.

4 Saravanan P, Davidson NC, Schmidt EB, Calder PC. Cardiovascular effects of marine omega-3 fatty acids. Lancet 2010;376:540-50.

5 Burillo E, Martin-Fuentes P, Mateo-Gallego R, Baila-Rueda L, Cenarro A, Ros E, et al. Omega-3 fatty acids and HDL. How do they work in the prevention of cardiovascular disease? Curr Vasc Pharmacol 2012;10:432-41.

6 Mozaffarian D, Wu JH. Omega-3 fatty acids and cardiovascular disease: effects on risk factors, molecular pathways, and clinical events. J Am Coll Cardiol 2011;58:2047-67. Kris-Etherton PM, Harris WS, Appel LJ. Fish consumption, fish oil, omega-3 fatty acids, and cardiovascular disease. Circulation 2002;106:2747-57.

8 NICE: Secondary prevention in primary and secondary care for patients following a myocardial infarction. May 2007. 2012. www.nice.org.uk/nicemedia/pdf/ CG48NICEGuidance.pdf.

9 He K. Fish, long-chain omega-3 polyunsaturated fatty acids and prevention of cardiovascular disease - eat fish or take fish oil supplement? Prog Cardiovasc Dis 2009;52:95-114.

10 Wang C, Harris WS, Chung M, Lichtenstein AH, Balk EM, Kupelnick B, et al. n-3 fatty acids from fish or fish-oil supplements, but not alpha-linolenic acid, benefit cardiovascular disease outcomes in primary- and secondary-prevention studies: a systematic review. Am J Clin Nutr 2006;84:5-17.

11 Marik PE, Varon J. Omega-3 dietary supplements and the risk of cardiovascular events: a systematic review. Clin Cardiol 2009;32:365-72.

12 Bosch J, Gerstein HC, Dagenais GR, Diaz R, Dyal L, Jung H, et al. n-3 fatty acids and cardiovascular outcomes in patients with dysglycemia. N Engl J Med 2012;367:309-18.

13 Kromhout D, Giltay EJ, Geleijnse JM. n-3 fatty acids and cardiovascular events after myocardial infarction. N Engl J Med 2010;363:2015-26.

14 Mozaffarian D, Longstreth WT Jr, Lemaitre RN, Manolio TA, Kuller LH, Burke GL, et al. Fish consumption and stroke risk in elderly individuals: the cardiovascular health study. Arch Intern Med 2005;165:200-6.

15 Larsson SC, Virtamo J, Wolk A. Fish consumption and risk of stroke in Swedish women. Am J Clin Nutr 2011;93:487-93. 


\section{What is already known on this topic}

Consumption of fish and long chain omega 3 fatty acids has been associated with a reduced risk of coronary heart disease and sudden cardiac death

However, observational and experimental evidence supporting a similar benefit for cerebrovascular disease remain conflicting

\section{What this study adds}

Observational findings in this meta-analysis show that consumption of both fish and long chain omega 3 fatty acids may modestly reduce the risk of cerebrovascular diseases

However, currently available evidence from long chain omega 3 fatty acid supplementation trials, based on both primary and secondary prevention studies, do not support the observational evidence

The beneficial effect of fish intake on cerebrovascular risk might be mediated through a complex interplay among a wide range of nutrients commonly found in fish

16 He K, Rimm EB, Merchant A, Rosner BA, Stampfer MJ, Willett WC, et al. Fish consumption and risk of stroke in men. JAMA 2002;288:3130-6.

17 Myint PK, Welch AA, Bingham SA, Luben RN, Wareham NJ, Day NE, et al. Habitual fish consumption and risk of incident stroke: the European Prospective Investigation into Cancer (EPIC)-Norfolk prospective population study. Public Health Nutr 2006:9:882-8.

18 Yamagishi K, Iso H, Date C, Fukui M, Wakai K, Kikuchi S, et al. Fish, omega-3 polyunsaturated fatty acids, and mortality from cardiovascular diseases in a nationwide community-based cohort of Japanese men and women the JACC (Japan Collaborative Cohort Study for Evaluation of Cancer Risk) Study. J Am Coll Cardiol 2008;52:988-96.

19 Morris MC, Manson JE, Rosner B, Buring JE, Willett WC, Hennekens CH. Fish consumption and cardiovascular disease in the physicians' health study: a prospective study. Am J Epidemiol 1995;142:166-75.

20 Gillum RF, Mussolino ME, Madans $\mathrm{JH}$. The relationship between fish consumption and stroke incidence. The NHANES I Epidemiologic Follow-up Study (National Health and Nutrition Examination Survey). Arch Intern Med 1996;156:537-42.

21 Montonen J, Jarvinen R, Reunanen A, Knekt P. Fish consumption and the incidence of cerebrovascular disease. Br J Nutr 2009:102:750-6.

22 He K, Song Y, Daviglus ML, Liu K, Van HL, Dyer AR, et al. Fish consumption and incidence of stroke: a meta-analysis of cohort studies. Stroke 2004;35:1538-42.

23 Hooper L, Thompson RL, Harrison RA, Summerbell CD, Ness AR, Moore HJ, et al. Risks and benefits of omega 3 fats for mortality, cardiovascular disease, and cancer: systematic review. BMJ 2006;332:752-60.

24 Larsson SC, Orsini N. Fish consumption and the risk of stroke: a dose-response meta-analysis. Stroke 2011:42:3621-3.

25 Stroup DF, Berlin JA, Morton SC, Olkin I, Williamson GD, Rennie D, et al. Meta-analysis of observational studies in epidemiology: a proposal for reporting. Meta-analysis Of Observational Studies in Epidemiology (MOOSE) group. JAMA 2000;283:2008-12.

26 Von Elm E, Altman DG, Egger M, Pocock SJ, Gotzsche PC, Vandenbroucke JP. The Strengthening the Reporting of Observational Studies in Epidemiology (STROBE) statement: guidelines for reporting observational studies J Clin Epidemiol 2008:61:344-9.

27 Wong WC, Cheung CS, Hart GJ. Development of a quality assessment tool for systematic reviews of observational studies (QATSO) of HIV prevalence in men having sex with men and associated risk behaviours. Emerg Themes Epidemiol 2008;5:23

28 Danesh J, Collins R, Appleby P, Peto R. Association of fibrinogen, C-reactive protein, albumin, or leukocyte count with coronary heart disease: meta-analyses of prospective studies. JAMA 1998:279:1477-82.

29 Greenland S, Longnecker MP. Methods for trend estimation from summarized dose-response data, with applications to meta-analysis. Am J Epidemiol 1992;135:1301-9.

30 Higgins JP, Thompson SG, Deeks JJ, Altman DG. Measuring inconsistency in meta-analyses. BMJ 2003;327:557-60.

31 Egger M, Davey SG, Schneider M, Minder C. Bias in meta-analysis detected by a simple, graphical test. BMJ 1997;315:629-34

32 Pilz S, Tomaschitz A, Drechsler C, Zittermann A, Dekker JM, Marz W. Vitamin D supplementation: a promising approach for the prevention and treatment of strokes. Curr Drug Targets 2011;12:88-96.

33 He K, Merchant A, Rimm EB, Rosner BA, Stampfer MJ, Willett WC, et al. Folate, vitamin $B 6$, and B12 intakes in relation to risk of stroke among men. Stroke 2004:35:169-74.

34 Militante JD, Lombardini JB. Treatment of hypertension with oral taurine: experimental and clinical studies. Amino Acids 2002:23:381-93.

35 Yamori Y, Horie R, Ohtaka M, Nara Y, Ikeda K. Prophylactic trials for stroke in stroke-prone SHR. (3) Amino acid analysis of various diets and their prophylactic effect. Jpn Heart $J$ 1978;19:624-6.
36 Moncada S, Higgs A. The L-arginine-nitric oxide pathway. N Engl J Med 1993;329:2002-12. 37 Hansen JC, Pedersen HS, Mulvad G. Fatty acids and antioxidants in the Inuit diet. Their role in ischemic heart disease (IHD) and possible interactions with other dietary factors. A review. Arctic Med Res 1994;53:4-17.

38 Van Mierlo LA, Arends LR, Streppel MT, Zeegers MP, Kok FJ, Grobbee DE, et al. Blood pressure response to calcium supplementation: a meta-analysis of randomized controlled trials. J Hum Hypertens 2006;20:571-80.

39 Amighi J, Sabeti S, Schlager O, Mlekusch W, Exner M, Lalouschek W, et al. Low serum magnesium predicts neurological events in patients with advanced atherosclerosis. Stroke 2004;35:22-7.

40 Ford ES. Serum magnesium and ischaemic heart disease: findings from a national sample of US adults. Int J Epidemiol 1999;28:645-51.

41 D'Elia L, Barba G, Cappuccio FP, Strazzullo P. Potassium intake, stroke, and cardiovascular disease a meta-analysis of prospective studies. J Am Coll Cardio 2011;57:1210-9.

42 Hoption Cann SA. Hypothesis: dietary iodine intake in the etiology of cardiovascular disease. J Am Coll Nutr 2006;25:1-11.

43 Bernstein AM, Pan A, Rexrode KM, Stampfer M, Hu FB, Mozaffarian D, et al. Dietary protein sources and the risk of stroke in men and women. Stroke 2012;43:637-44.

44 Hu FB, Bronner L, Willett WC, Stampfer MJ, Rexrode KM, Albert CM, et al. Fish and omega-3 fatty acid intake and risk of coronary heart disease in women. JAMA 2002;287:1815-21.

45 Candela M, Astiasaran I, Bello J. Deep-fat frying modifies high-fat fish lipid fraction. J Agric Food Chem 1998;46:2793-6.

46 US Department of Agriculture. Composition of foods raw, processed, prepared USDA national nutrient database for standard reference, release 24. Sept 2011. 2012. www.ars. usda.gov/SP2UserFiles/Place/12354500/Data/SR24/sr24_doc.pdf.

47 Harris K, Felming J, Kris-Etherton P. Challenges in estimating omega-3 fatty acid content of seafood from US nutrient databases: a salmon case study. J Food Compost Anal 2011;24:1168-73.

48 USDA Nutrient Data Laboratory. 2012. www.ars.usda.gov/main/site_main.htm? modecode $=12-35-45-00$

49 Welch AA, Bingham SA, Ive J, Friesen MD, Wareham NJ, Riboli E, et al. Dietary fish intake and plasma phospholipid $\mathrm{n}-3$ polyunsaturated fatty acid concentrations in men and women in the European Prospective Investigation into Cancer-Norfolk United Kingdom cohort. Am J Clin Nutr 2006:84:1330-9.

50 Hodson L, Skeaff CM, Fielding BA. Fatty acid composition of adipose tissue and blood in humans and its use as a biomarker of dietary intake. Prog Lipid Res 2008;47:348-80.

51 Mozaffarian D, Ludwig DS. Dietary guidelines in the 21st century-a time for food. JAMA 2010;304:681-2.

Accepted: 24 September 2012

\section{Cite this as: BMJ 2012;345:e6698}

This is an open-access article distributed under the terms of the Creative Commons Attribution Non-commercial License, which permits use, distribution, and reproduction in any medium, provided the original work is properly cited, the use is non commercial and is otherwise in compliance with the license. See: $\mathrm{http}: / /$ creativecommons.org/licenses/by$\mathrm{nc} / 2.0 /$ and http://creativecommons.org/licenses/by-nc/2.0/legalcode. 


\section{Table}

Table 1| Summary of data included in systematic review

\begin{tabular}{|c|c|c|c|c|}
\hline Study types & No of studies & Total No of participants & Average follow-up (years) & $\begin{array}{c}\text { No of incident cerebrovascular } \\
\text { events }\end{array}$ \\
\hline \multicolumn{5}{|l|}{ Prospective cohort studies } \\
\hline All studies*: & 26 & 731960 & 15.0 & 26569 \\
\hline Fish & 21 & 675048 & 15.1 & 25320 \\
\hline Long chain omega 3 fatty acids & 14 & 305119 & 13.0 & 5374 \\
\hline $\begin{array}{l}\text { RCTs of long chain omega } 3 \text { fatty acid } \\
\text { supplementation }\end{array}$ & 12 & $62040 \dagger$ & 3.0 & $1563 \ddagger$ \\
\hline Total unique studies & 38 & 794000 & & 34817 \\
\hline
\end{tabular}

General characteristics of individual studies are described in detail in the supplementary tables.

$\mathrm{RCT}=$ randomised controlled trial.

*Nine studies reported on consumption of both fish and long chain omega 3 fatty acids.

†Includes 31088 and 30952 total participants in intervention and control groups, respectively.

fIncludes 800 and 763 cerebrovascular disease events in intervention and control groups, respectively. 


\section{Figures}

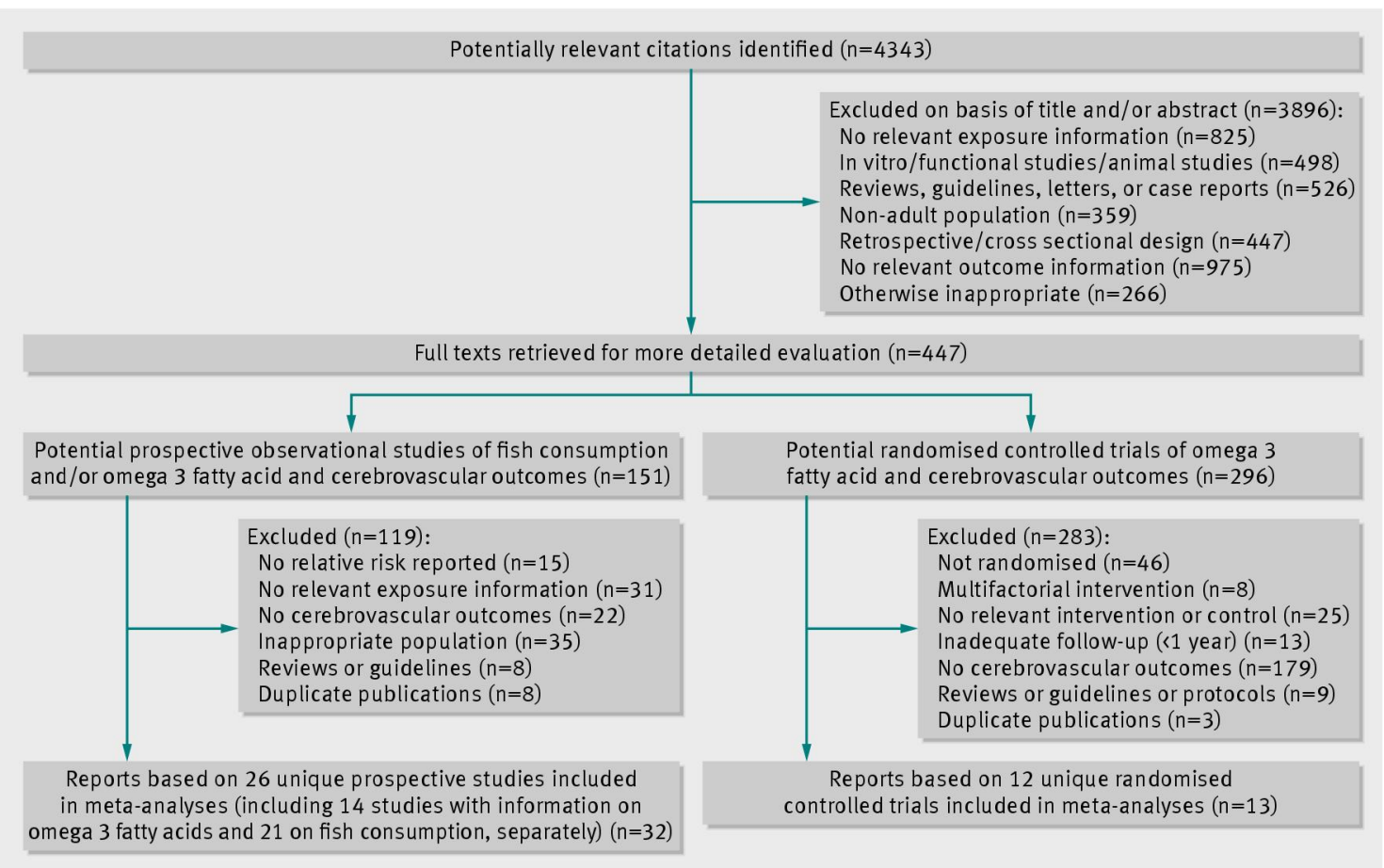

Fig 1 Flow of studies in review

\begin{tabular}{|c|c|c|c|c|c|}
\hline \multirow{2}{*}{ Categories of fish intake } & $\begin{array}{c}\text { No of } \\
\text { studies }\end{array}$ & $\begin{array}{c}\text { No of } \\
\text { participants }\end{array}$ & $\begin{array}{l}\text { No of } \\
\text { events }\end{array}$ & $\begin{array}{c}\text { Relative risk } \\
(95 \% \mathrm{Cl})\end{array}$ & \multirow[t]{2}{*}{$\begin{array}{c}\text { Relative risk } \\
(95 \% \mathrm{CI})\end{array}$} \\
\hline & & & & & \\
\hline $2-4 v \leq 1$ servings/week & 18 & 650210 & 24612 & $\rightarrow$ & 0.94 (0.90 to 0.98$)$ \\
\hline$\geq 5 v \leq 1$ servings/week & 8 & 394958 & 16890 & $\rightarrow-$ & 0.88 (0.81 to 0.96$)$ \\
\hline \multicolumn{6}{|l|}{ Dose-response assessment } \\
\hline Increment of 2 servings/week & ek 18 & 669727 & 24791 & $\rightarrow$ & 0.96 (0.93 to 0.99$)$ \\
\hline & & & & 0.9 & \\
\hline
\end{tabular}

Fig 2 Association between fish consumption and risk of cerebrovascular disease in prospective cohort studies with information on intake categories and weekly increment of servings

\begin{tabular}{|c|c|c|c|c|c|}
\hline Long chain omega 3 fatty acids & $\begin{array}{l}\text { No of } \\
\text { studies } \\
\text { s }\end{array}$ & $\begin{array}{l}\text { No of } \\
\text { participants }\end{array}$ & $\begin{array}{l}\text { No of } \\
\text { events }\end{array}$ & $\begin{array}{l}\text { Relative risl } \\
\quad(95 \% \mathrm{Cl})\end{array}$ & $\begin{array}{l}\text { Relative risk } \\
\quad(95 \% \mathrm{CI})\end{array}$ \\
\hline All cohort studies & 14 & 305119 & 5374 & $\rightarrow$ & $0.94(0.86$ to 1.02$)$ \\
\hline Circulating biomarker studies & s 4 & 4096 & 1177 & & $1.04(0.90$ to 1.20$)$ \\
\hline \multirow[t]{3}{*}{ Dietary studies } & 10 & 301023 & 4197 & $\rightarrow$ & $0.90(0.80$ to 1.01$)$ \\
\hline & 4 & 201551 & 1141 & & $0.85(0.70$ to 1.03$)$ \\
\hline & 4 & 201551 & 710 & & $0.77(0.59$ to 1.01$)$ \\
\hline \multicolumn{6}{|l|}{ Fish consumption } \\
\hline & 11 & 366787 & 11349 & 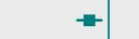 & 0.91 (0.86 to 0.97$)$ \\
\hline & 7 & 284178 & 5002 & $\rightarrow-$ & 0.93 (0.87 to 0.99$)$ \\
\hline & 7 & 284178 & 1783 & $\multimap$ & 0.81 (0.70 to 0.94$)$ \\
\hline Any cerebrovascular disease & & & & 0.751 & \\
\hline
\end{tabular}

$\square$ Ischaemic stroke

- Haemorrhagic stroke

Fig 3 Association of long chain omega 3 fatty acids and fish consumption with cerebrovascular disease, based on prospective cohort studies, comparing risk in top versus bottom third of baseline levels. *To allow consistent indirect comparison, includes prospective cohort studies with available risk estimates comparing top versus bottom thirds of fish consumption 


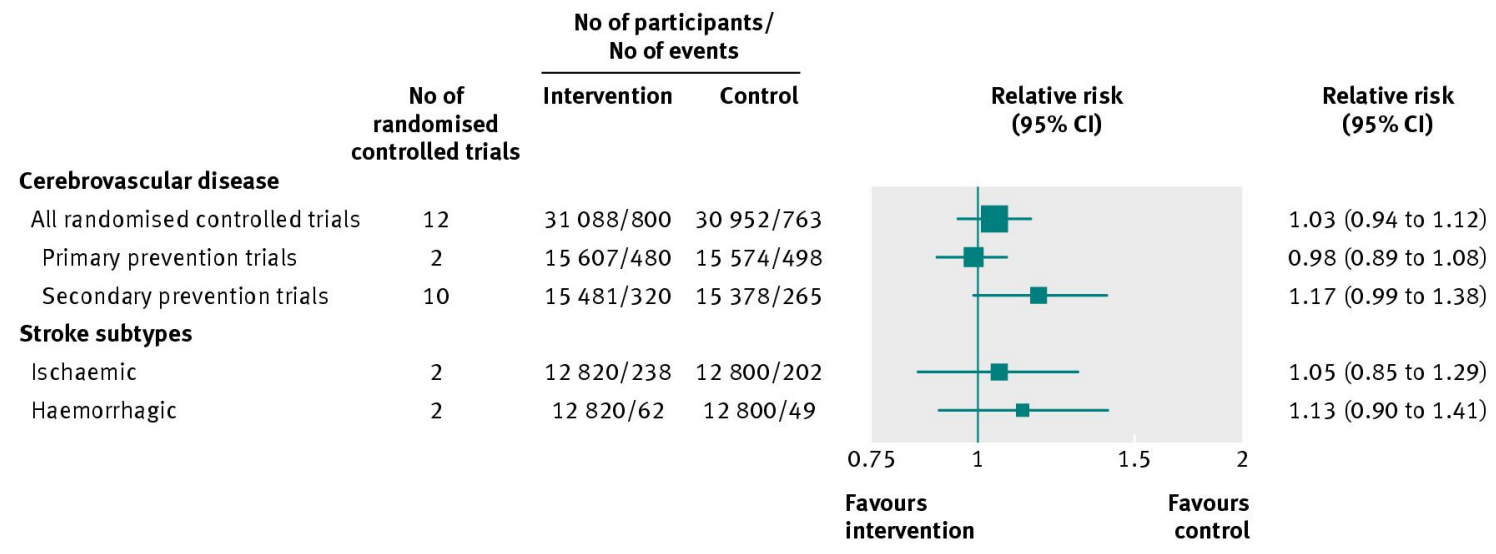

Fig 4 Effects of long chain omega 3 fatty acid supplementations on cerebrovascular disease, derived from randomised control trials. *Trials involving participants with no pre-existing cardiovascular disease at baseline. $†$ Trials including participants with previous or existing cardiovascular disease at baseline. ‡Based on studies with available information on subtype 